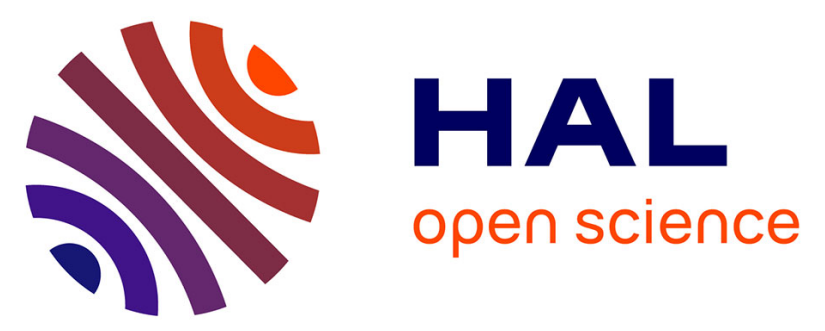

\title{
Contribution à l'étude du littoral de la baie d'Audierne (Finistère). Nature et origine des minéraux lourds des plages
}

Louis Chauris

\section{- To cite this version: \\ Louis Chauris. Contribution à l'étude du littoral de la baie d'Audierne (Finistère). Nature et origine des minéraux lourds des plages. Norois, 1992, 39 (156), pp.451-458. 10.3406/noroi.1992.6449 . insu- 01860956}

\section{HAL Id: insu-01860956 \\ https://hal-insu.archives-ouvertes.fr/insu-01860956}

Submitted on 3 Feb 2021

HAL is a multi-disciplinary open access archive for the deposit and dissemination of scientific research documents, whether they are published or not. The documents may come from teaching and research institutions in France or abroad, or from public or private research centers.
L'archive ouverte pluridisciplinaire HAL, est destinée au dépôt et à la diffusion de documents scientifiques de niveau recherche, publiés ou non, émanant des établissements d'enseignement et de recherche français ou étrangers, des laboratoires publics ou privés. 


\section{Contribution à l'étude du littoral de la baie d'Audierne (Finistère). Nature et origine des minéraux lourds des plages}

\section{Louis Chauris}

\section{Résumé}

RÉSUMÉ

L'étude des minéraux lourds concentrés sur l'estran de la baie d'Audierne indique qu'un pourcentage important des sables littoraux présente une origine distale. Ces résultats sont en bon accord avec les données déduites antérieurement de l'examen des galets du cordon littoral.

\section{Abstract ABSTRACT}

The study of heavy minerals concentrated along the beaches of the Audierne bay points out that an important percentage of the coastal sands presents a distal origin. These results agree with the data inferred from the first investigations on the pebbles of the coastal strand.

\section{Citer ce document / Cite this document :}

Chauris Louis. Contribution à l'étude du littoral de la baie d'Audierne (Finistère). Nature et origine des minéraux lourds des plages. In: Norois, n¹56, Octobre-Décembre 1992. pp. 451-458;

doi : https://doi.org/10.3406/noroi.1992.6449

https://www.persee.fr/doc/noroi_0029-182x_1992_num_156_1_6449

Fichier pdf généré le 10/11/2019 
Norois, 1992, Poitiers, t. 39, no 156, p. 451-458

\title{
CONTRIBUTION A L'ÉTUDE DU LITTORAL DE LA BAIE D'AUDIERNE (Finistère) NATURE ET ORIGINE DES MINÉRAUX LOURDS DES PLAGES
}

\author{
par Louis CHAURIS
}

Département des Sciences de la Terre, URA 1278

Université de Bretagne occidentale, 29287 Brest

\section{RÉSUMÉ}

L'étude des minéraux lourds concentrés sur l'estran de la baie d'Audierne indique qu'un pourcentage important des sables littoraux présente une origine distale. Ces résultats sont en bon accord avec les données déduites antérieurement de l'examen des galets du cordon littoral.

\section{$A B S T R A C T$}

The study of heavy minerals concentrated along the beaches of the Audierne bay points out that an important percentage of the coastal sands presents a distal origin. These results agree with the data inferred from the first investigations on the pebbles of the coastal strand.

"Devant la mer seule, ou presque."

A. Guilcher, 1948.

La baie d'Audierne reste encore l'un des secteurs les plus attachants et les plus étudiés des côtes finistériennes. Le bruit et la fureur des éléments lors des tempêtes hivernales, les brumes ouatées dérobant le rivage au regard... ont paru stimuler les recherches! En particulier, l'immense cordon de galets - l'Ero Vili des Bretons a souvent attiré l'attention depuis E. de Fourcy (1844) et E. de Beaumont (1845). Les anciennes accumulations de galets et leur âge, les sables de l'estran et des dunes, le recul spectaculaire du trait de côte... ont fait l'objet de nombreuses investigations, parmi lesquelles il faut citer les travaux de Barrois $(1876,1882)$, Guilcher (1948), Toulemont (1964), Guilcher et Giot (1969), Bodéré (1971), Guilcher et Hallégouët (1981), Guilcher (1982) pour se limiter aux œuvres les plus significatives.

Mots clés: Minéraux lourds. Sables littoraux. Baie d’Audierne. Massif armoricain.

Key words: Heavy minerals. Coastal sands. Audierne bay. Armorican Massif. 
L'un des problèmes les plus intéressants est celui de l'origine des galets du célèbre cordon. Un certain pourcentage a une source locale et provient, à l'évidence, des basses falaises littorales attaquées par la mer (micaschistes et amphibolites de Penhors...) (Bodéré, 1971). Les aplites sont beaucoup plus rares [la "leptynite»" des auteurs est en fait une aplite albitique (Jeanneau, 1967; Chauris, 1974)]. L'apport par les ruisseaux côtiers est ici très mineur (Guilcher, 1948). Dans une forte proportion, les matériaux sont indubitablement d'origine lointaine: c'est le cas, en particulier, des microgranites, si abondants, et des silex, subordonnés. L'apport par les glaces flottantes a ses adeptes depuis Barrois (1876) - et ici les sources peuvent être très distales. On sait depuis peu que les basaltes éparpillés sur les estrans de Bretagne occidentale proviennent d'Islande (Bellon et al., 1988). Les éléments arrachés au plateau continental ont joué aussi un rôle non négligeable: c'est le cas pour les silex, confirmé par la découverte du Crétacé au large du littoral (Saint-Requier, 1970). Au total, pour les galets, les sources "marines" l'emportent sur les sources «continentales».

Un problème de provenance se pose aussi pour les sables de l'estran : sporadiques - comme les accumulations de galets - le long de la côte rocheuse au nord de Penhors (secteur I) ; largement étendus au pied du cordon de galets, entre Penhors et les environs de Tréguennec (secteur II); prédominants à la base des accumulations dunaires, de Tréguennec à la pointe de la Torche (secteur III) (fig. 1). Pour tenter, sinon de résoudre cette question, tout au moins de la circonscrire, 14 échantillons ont été prélevés sur l'estran, par raclage à la pelle de zones minéralisées en sables lourds (petits placers littoraux) et analysés selon la méthode préconisée au B.R.G.M. (Guigues et Devismes, 1969). Notre objectif était triple: 1) obtenir des informations sur l'origine - proximale ou distale — des sables de l'estran par l'examen des concentrés de minéraux lourds [comme l'examen pétrographique des galets avait permis de faire la part des sources locales et lointaines de ce matériau (Guilcher, 1948 ; Bodéré, 1971)] ; 2) confronter les données déduites de l'étude minéralogique des sables avec celles recueillies antérieurement par les comptages de galets (Bodéré, 1971); 3) compléter ainsi les connaissances sur les dépôts littoraux de la baie d'Audierne. Les résultats des analyses minéralogiques sont présentés dans le tableau I et synthétisés sur les figures 1 à 6 .

\section{I. - LOCALISATION ET MORPHOLOGIE DES ACCUMULATIONS DE MINÉRAUX LOURDS}

Dans l'ensemble, les concentrations restent sporadiques. Cinq types morphologiques ont été distingués. 1) Au pied même du cordon de galets, à la partie supérieure des sables de l'estran, placages superficiels et minces couches en profondeur, sur des extensions longitudinales qui peuvent dépasser 100 mètres (cas fréquent). 2) Dans les zones sableuses des croissants des plages qui accidentent le cordon de galets (Crumuni). 3) Dans la plage ancienne sableuse qui apparaît parfois sur l'estran, sous le cordon de galets actuel. 4) Au pied des dunes, toujours en haut d'estran, sur 5 à 10 mètres de large et plus de 100 mètres de long (ouest de Tronoën...). 5) Dans le lit des ruisselets parcourant, à basse mer, la partie haute sableuse de l'estran; ces ruisselets qui apparaissent en particulier au débouché des étangs littoraux barrés par le cordon de galets, sont dénommés "revorennou" (Guilcher, 1948). 

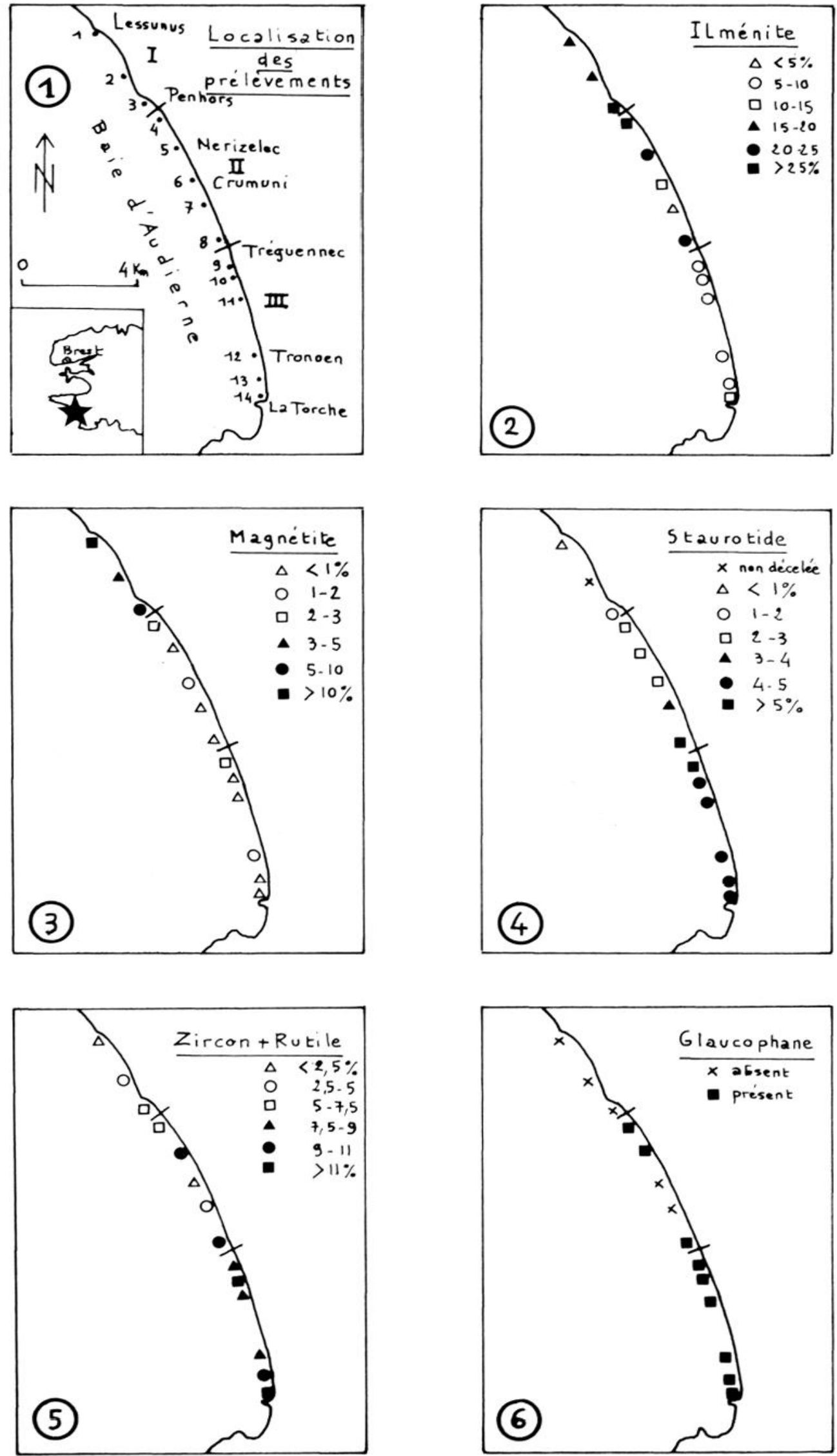

FIG. 1 à 6. - Localisation des prélèvements de sables lourds et pourcentages (en poids) de quelques minéraux lourds de la baie d'Audierne (voir tableau I). 
TABLEAU 1. - Minéraux lourds des petits placers littoraux de la baie d'Audierne. I à III. Secteurs distingués. 1 à 14 . Localisation des prélèvements sur la figure $1 . \%$ M.L.: Pourcentage des minéraux lourds par rapport au sable brut recueilli par raclage à la pelle. Pourcentage des différents M.L. en poids. - : présent ; tr : traces; mtr : microtraces. (Analyses H. Chevance).

\begin{tabular}{|c|c|c|c|c|c|c|c|c|c|c|c|c|c|c|}
\hline & \multicolumn{3}{|c|}{ I } & \multicolumn{5}{|c|}{ II } & \multicolumn{6}{|c|}{ III } \\
\hline & 1 & 2 & 3 & 4 & 5 & 6 & 7 & 8 & 9 & 10 & 11 & 12 & 13 & 14 \\
\hline$\%$ M.L. & 42,7 & 26,9 & 46,3 & 11,7 & 10,2 & 75,2 & 36,6 & 14,0 & 27,8 & 21,8 & 20,9 & 34,5 & 25,5 & 23,4 \\
\hline Zircon & 1,4 & 2,7 & 5,1 & 4,3 & 4,6 & 1,3 & 1,7 & 4,6 & 5,6 & 5,5 & 5,0 & 6,0 & 6,3 & 5,8 \\
\hline Tourmaline & - & - & - & 2,2 & 3,1 & 1,8 & 7,3 & 6,1 & 2,1 & 5,5 & 7,1 & 4,1 & 4,9 & 7,1 \\
\hline Rutile & 0,7 & 1,3 & 1,9 & 2,1 & 5,4 & 0,9 & 1,7 & 4,6 & 2,8 & 5,6 & 3,5 & 2,7 & 4,2 & 5,7 \\
\hline Anatase & - & - & - & - & - & - & & - & - & - & - & - & - & - \\
\hline Monazite & 1,1 & $\operatorname{tr}$ & $\mathrm{mtr}$ & $\mathrm{mtr}$ & $\mathrm{mtr}$ & $\mathrm{mtr}$ & $\mathrm{mtr}$ & $\mathrm{mtr}$ & $\mathrm{mtr}$ & $\mathrm{mtr}$ & & $\mathrm{mtr}$ & $\mathrm{mtr}$ & \\
\hline Corindon & - & - & - & - & & - & - & - & - & - & & - & - & - \\
\hline Scheelite & $\operatorname{tr}$ & & $\mathrm{mtr}$ & & & $\mathrm{mtr}$ & & & & & & & & \\
\hline Ilménite & 18,8 & 16,2 & 30,4 & 28,2 & 20,1 & 11,6 & 3,9 & 24,4 & 9,1 & 7,4 & 8,5 & 6,8 & 9,1 & 14,3 \\
\hline Magnétite & 20,3 & 4,0 & 5,0 & 2,2 & 0,8 & 1,1 & 0,8 & 0,7 & 2,1 & - & 0,7 & 1,3 & 0,7 & 0,5 \\
\hline Staurotide & - & & 1,3 & 2,1 & 2,3 & 2,2 & 3,8 & 6,1 & 5,6 & 4,4 & 5,0 & 4,9 & 4,2 & 4,8 \\
\hline Andalousite & - & - & - & - & - & 0,3 & 1,3 & & - & - & & 0,8 & - & - \\
\hline Sillimanite & - & - & - & & - & - & & & - & & & - & - & \\
\hline Disthène & - & - & - & - & - & - & - & - & - & - & - & - & - & - \\
\hline Grenat & 52,2 & 66,2 & 50,0 & 30,4 & 40,2 & 78,1 & 78,0 & 30,5 & 56,0 & 51,1 & 51,9 & 60,1 & 62,4 & 47,8 \\
\hline Epidote & 2,1 & 5,4 & 5,1 & 10,8 & 18,6 & 0,9 & - & 15,2 & 14,1 & 14,8 & 12,8 & 11,7 & 4,2 & 11,9 \\
\hline Amphibole & 2,1 & 2,7 & - & 15,2 & 3,9 & 0,2 & - & 6,9 & 1,4 & 4,6 & 4,2 & 0,3 & 2,8 & - \\
\hline Pyroxène & - & & & - & & - & - & & & & & & - & \\
\hline Apatite & - & - & - & - & - & & - & & - & - & - & & - & - \\
\hline Sphène & & - & - & - & - & & & & - & - & & & & - \\
\hline Spinelle & & & & & & - & & & & & & & & \\
\hline Pyrite & - & - & - & - & & & & & & & - & & - & \\
\hline Mispickel & - & & & - & & & & & & & & & & \\
\hline Barytine & & & $\mathrm{mtr}$ & & & & & & & & & & & \\
\hline Topaze & & & & & & - & & & & & & & & \\
\hline Or & & & & & & $\mathrm{mtr}$ & $\mathrm{mtr}$ & & & & & & $m k r$ & \\
\hline Glaucophane & & & & - & - & & & - & - & - & - & - & - & - \\
\hline
\end{tabular}

\section{II. - COMPOSITION MINÉRALOGIQUE}

Les fortes concentrations en minéraux lourds (M.L.) restent exceptionnelles. La teneur en M.L. par rapport au sable brut recueilli, varie selon les points, de $10 \%$ (débouché de l'étang de Nérizelec) à $75 \%$ (Crumuni). La teneur moyenne est plus élevée dans le secteur I $(38,6 \%)$ que dans les secteurs II $(29,5 \%)$ et III $(25,6 \%)$. Au total, 26 M.L. différents ont pu être identifiés. Le spectre est dominé - et de loin - par le grenat $(53,9 \%)$. Viennent ensuite l'ilménite $(14,9 \%)$, l'épidote $(9,1 \%)$, le zircon $(4,3 \%)$, la tourmaline $(3,7 \%)$, la staurotide $(3,3 \%)$, l'amphibole et le rutile 
$(3,1 \%)$, enfin la magnétite $(2,9 \%)$. Toutes les autres espèces ont des teneurs moyennes $<1 \%$ (tableau 1 ). L'abondance du grenat confère, le plus souvent, aux petits placers, une coloration rougeâtre; toutefois, vers le nord, la magnétite et l'ilménite tendent à leur donner une nuance noirâtre (Lessunus).

\section{III. - RÉPARTITION DES MINÉRAUX LOURDS SELON LES TROIS SECTEURS}

* Le secteur septentrional (I), à dominante rocheuse (3 échantillons) se caractérise par sa teneur moyenne assez élevée en magnétite $(9,7 \%)$ et en ilménite $(21,8 \%)$, la présence significative de monazite (un prélèvement à $1,1 \%$ ), un peu de scheelite (2 éch. sur 3) et même de mispickel (1/3); inversement, les teneurs sont relativement faibles en rutile $(1,3 \%)$, épidote $(4,2 \%)$, amphibole $(1,6 \%)$ et surtout en staurotide $(0,4 \%)$. Le glaucophane est totalement absent. Dans l'échantillon le plus septentrional (Lessunus), la magnétite présente encore de nombreux octaèdres aux formes cristallines parfaites, lilménite est souvent peu émoussée, le grenat très esquilleux. Cette absence générale d'usure s'attẻnue progressivement vers le sud (Penhors).

* Le secteur médian (II), correspondant essentiellement à l'Ero Vili (5 éch.), se signale par la chute des teneurs moyennes en magnétite $(1,1 \%$ seulement), la décroissance du pourcentage en ilménite $(17,6 \%)$, la rareté de la scheelite (dans un seul échantillon) et de la monazite (uniquement en microtraces), l'augmentation des teneurs en rutile $(2,9 \%)$, staurotide $(3,3 \%)$, épidote $(9,1 \%)$ et amphibole $(5,2 \%)$. Le glaucophane est décelé dans trois prélèvements. Dans l'ensemble, par rapport au secteur I, on constate une augmentation très sensible de l'émoussé, qui peut être excellent.

* Le secteur méridional (III) coïncide avec la côte dunaire (6 éch.). Les teneurs moyennes en magnétite ont encore diminué $(0,9 \%)$, les teneurs en ilménite ont nettement chuté $(9,2 \%)$, la scheelite et le mispickel n'ont jamais été observés, la monazite peut manquer. Par contre, les teneurs moyennes en zircon sont ici singulièrement élevées $(5,7 \%)$; il en est de même pour le rutile $(4,1 \%)$. Les teneurs en staurotide $(4,8 \%)$ et en épidote $(11,6 \%)$ ont légèrement augmenté par rapport à celles du secteur II. Le glaucophane est décelé dans tous les prélèvements. Dans l'ensemble, l'émoussé est souvent excellent.

\section{IV. - SOURCE DES MINERAUX LOURDS}

Les données minéralogiques obtenues sur les sables lourds prélevés sur l'estran, confrontées avec les résultats des prospections alluvionnaires en lit vif effectuées par le B.R.G.M. (Devismes et Chevance, sans date) dans l'arrière-pays, et avec les cartes géologiques régionales (Barrois, 1891 ; Peucat, 1973), permettent de présenter plusieurs déductions importantes sur la provenance des minéraux lourds concentrés sur le littoral de la baie d'Audierne et, par suite, sur les sables qui les renferment. Sous cet aspect, les minéraux lourds peuvent être regroupés, en première approximation, sous trois rubriques.

\section{* Les minéraux d'origine locale.}

Ici se classent essentiellement la magnétite, la scheelite, la monazite et, pro parte, l'ilménite. La magnétite est abondante dans l'arrière pays, où elle est associée en particulier aux roches vertes. Toutefois, sur les estrans, elle se concentre uniquement dans le secteur où le socle est en contact direct avec le rivage. Cette 
localisation restreinte confirme pleinement les observations de Guilcher (1948) sur le faible apport des ruisseaux débouchant en baie d'Audierne. La même remarque peut être formulée pour la scheelite. La monazite n'apparaît de manière significative que dans la partie septentrionale de l'arrière-pays et c'est seulement sur le littoral adjacent que ses teneurs dépassent $1 \%$. L'ilménite, en liaison avec les roches basiques de Peumerit, est abondante dans la moitié septentrionale de l'arrièrepays; elle est, par contre, rare dans la moitié méridionale (leucogranites de PlonéourLanvern et de Pont-l'Abbé). Ainsi peut s'interpréter, au moins en partie, la nette décroissance des teneurs sur les estrans méridionaux de la baie d'Audierne. $\mathrm{Ce}$ minéral forme transition avec le groupe suivant.

\section{* Ies minéraux d'origine mixte.}

A ce groupe appartient tout d'abord le grenat. Ce minéral ne présente pas de variations significatives dans les trois secteurs étudiés (respectivement 56,1,51,4 et $54,9 \%$ ). Il est abondant dans la partie septentrionale de l'arrière-pays, mais aussi sur la plate-forme continentale (Lapierre, sans date). Les analyses effectuées le long des côtes méridionales de la Bretagne (Chauris, 1982, 1987) soulignent sa fréquence dans les placers littoraux. Il représente le principal minéral lourd du stock étalé sur la plate-forme littorale et déplacé au cours des épisodes de transgressions et régressions. Son origine est ici pour une part importante certainement distale. La répartition de l'épidote soulève, à première vue, un problème assez difficile. Ce minéral est omniprésent dans les alluvions fluviatiles de l'arrière-pays, tant dans le domaine des roches vertes (Peumerit) - ce qui est normal - que dans celui des leucogranites (Plonéour-Lanvern, Pont-l'Abbé) - qui n'en contiennent pas. Sa présence dans la région granitique s'expliquerait au mieux par les transgressions quaternaires qui l’ont étalé sur toute la pénéplaine du pays bigouden, sans être influencées nécessairement par la nature du substratum. Ses sources sont partiellement distales.

\section{* Les minéraux d'origine distale prépondérante.}

La staurotide n'est pas décelée dans l'arrière-pays. Sur l'estran, les seules teneurs moyennes relativement élevées sont notées dans les secteurs II $(3,3 \%)$ et III $(4,8 \%)$, alors que le minéral est rare, voire absent, dans le secteur I. Les teneurs moyennes en zircon + rutile, minéraux très résistants, sont singulièrement élevées dans les secteurs II $(6,2 \%)$ et surtout III $(9,8 \%)$, qui s'opposent ainsi au secteur I $(4,3 \%)$. C'est dans le secteur III qu'ont été décelées les plus fortes teneurs en l'association zircon + rutile de toutes les côtes bretonnes (sur environ 500 prélèvements [Chauris, travaux en cours]). A première vue, ces pourcentages élevés pourraient être mis en relation avec les sévères conditions hydrodynamiques qui règnent sur la côte. Ces conditions auraient, en partie, détruit les M.L. moins résistants et ainsi peu à peu enrichi le cortège en espèces très résistantes. Sans rejeter entièrement cette manière de voir, on remarque que la présence, dans les mêmes prélèvements, de teneurs assez élevées en minéraux verts (épidote, amphibole) s'accorde difficilement avec cette interprétation. La rareté du zircon et du rutile dans l'arrière-pays suggère plutôt que les fortes teneurs décelées sur les estrans sont à attribuer à des sources lointaines (minéraux dispersés depuis longtemps sur la plate-forme continentale). L'absence totale de glaucophane dans l'arrière-pays indique à l'évidence son origine distale dans les sables de l'estran. Les occurrences les plus proches connues en Bretagne se situent nettement plus à l'est, à lîle de Groix, qui ne peut être, du fait de la dérive littorale, la source de ce minéral en baie d'Audierne.

De même que les galets du cordon actuel sont alimentés par le cordon ancien, une proportion sans doute importante des minéraux lourds décelés aujourd'hui sur l'estran provient des formations quaternaires. Les travaux de Toulemont (1964) 
ont noté dans ces dépôts la présence de staurotide, de zircon, de rutile, de glaucophane... Sables des plages et des dunes anciennes jouent ainsi le rôle de relais. Mais dans ces accumulations, la provenance d'une part importante des matériaux, pour les raisons évoquées plus haut, était déjà lointaine, en l'absence de modifications majeures des conditions morphologiques, climatiques et océanographiques.

\section{CONCLUSION}

L'étude des minéraux lourds de la baie d'Audierne établit qu'un pourcentage important - toutefois impossible à chiffrer — des sables de l'estran, est d'origine lointaine. Cette manière de voir est attestée en particulier par la staurotide, le zircon, le rutile, le glaucophane, ainsi que, pour partie, par l'ilménite, le grenat et l'épidote. Les données obtenues par l'examen des minéraux lourds sont en bon accord avec les déductions tirées antérieurement (Bodéré, 1971) de l'étude des galets de l'estran: pour une part significative, d'origine locale dans la zone nord de la baie (secteur I) et d'origine distale, ailleurs (secteurs II et III). Ainsi, deux approches totalement différentes aboutissent à des conclusions comparables - ce qui ne peut qu'appuyer le bien-fondé des deux méthodes.

\section{BIBLIOGRAPHIE}

BARRoIS (Ch.), 1876 - Note sur les traces de l'époque glaciaire en quelques points des côtes de Bretagne. Ann. Soc. géol. Nord., IV, p. 186-204.

BARrois (Ch.), 1882 - Sur les plages soulevées de la côte occidentale du Finistère. Ann. Soc. géol. Nord, IX, p. 239-268.

BARrols (Ch.), 1891 - Carte géologique à $1 / 80000$, feuille Quimper.

BEAUMONT (E. de), 1845 - Leçons de géographie pratique. T. I., $557 \mathrm{p}$.

Bellon (H.), Chauris (L.), Hallegouet (B.) et Thonon (P.), 1988 - Age et origine des roches volcaniques observées sur les estrans de l'extrême ouest du Massif armoricain. (France). Norois, T. 35, no 139, p. 331-335.

BodéRÉ (J.C.), 1971 - Observations sur la côte de la baie d'Audierne entre Penhors et Porz Carn. Cahiers océanographiques, XXIII, p. 519-543.

Chauris (L.), 1974 - Les leucogranites feuilletés des environs de Quimper (Massif armoricain) et leurs minéralisations en béryllium, lithium, étain et tungstène. Bull. Soc. géol. minéral. Bretagne, C, VI, 2, p. 91-102.

Chauris (L.), 1982 - Les sables lourds des plages du Mor Bras. Introduction à l'étude des placers littoraux en Bretagne méridionale. Bull. Soc. Sc. nat. Ouest de la France, nvelle sér., T. 4, p. 1-58.

Chauris (L.), 1987 - Composition minéralogique et origine des placers littoraux dans quatre îles du Morbihan. Géologie de la France, I, p. 55-63.

Devismes (P.) et Chevance (H.) (sans date). Atlas à $1 / 50000$ des minéraux d'alluvions du Massif armoricain. B.R.G.M., inédit.

Fourcy (E. de), 1844. Explication de la carte géologique du Finistère. Paris, 196 p.

Guigues (J.) et Devismes (P.), 1969 - La prospection minière à la batée dans le Massif armoricain. Mém. B.R.G.M., no 71,172 p.

Guilcher (A.), 1948 -- Le relief de la Bretagne méridionale de la baie de Douarnenez à la Vilaine. 682 p. La Roche-sur-Yon, Impr. Edit. H. Potier.

GUilcher (A.), 1982 - Remarques sur l'âge des cordons de galets anciens de la baie d'Audierne. Bull. Ass. Fr. Etud. Quaternaire, p. 205-206.

Gullcher (A.) et Giot (P.R.), 1969. Livret-guide de l'excursion C 16 Bretagne-Anjou, $V I I I^{e}$ Congr. INQUA, $79 \mathrm{p}$. 
Guilcher (A.) et Hallegouet (B.), 1981 - Le haut cordon de galets pléistocène de Ruvein en Plovan (Finistère) et ses enseignements généraux. Bull. Ass. fr. Etud. Quaternaire, 18, p. 75-82.

JEANNEAU (J.Y.), 1967 - Etude géologique et métallogénique des roches vertes de la baie d'Audierne. Thèse $3^{\text {ème }}$ cycle, Univers. Paris, 87 p., 24 pl.

Peucat (J.J.), 1973 - Les schistes cristallins de la baie d'Audierne. Etude pétrographique et structurale. Thèse $3^{\text {teme }}$ cycle, Univers. Rennes, $114 \mathrm{p}$.

SAINT-Requier (A.), 1970 - La baie d'Audierne. Etude de morphologie et de sédimentologie sous-marines. Thèse $3^{\text {ème }}$ cycle, Univers. Paris, $223 \mathrm{p}$.

TOULEMONT (M.), 1964 - Contribution à l'étude sédimentologique du Quaternaire du littoral sud-finistérien de la pointe du Raz à l'estuaire de l'Odet. Thèse 3ème cycle, Univers. Paris, 113 p. 